\title{
A Study Demographic, Histopathological Patterns and Clinical Profile of Oral Squamous Cell Carcinoma in East Java, Indonesia: A Hospital-Based Study
}

\section{Kania Difa Parama ${ }^{1,2 *}$, Nina Irawati ${ }^{3}$, Sunarto Reksoprawiro ${ }^{3}$, Sahudi $^{3}$}

${ }^{1}$ Specialized Residency Training, Department of General Surgery, Faculty of Medicine, Universitas Airlangga, Surabaya, Indonesi a 2Department of Surgery, Dr. Soetomo General Hospital, Surabaya, Indonesia

${ }^{3}$ Head and Neck Surgery Unit, Department of Surgery, Dr. Soetomo General Hospital, Surabaya, Indonesia

\section{A R T I C L E I N F O}

Keywords:

Oral cancer

Indonesia

Tobacco use

Alcohol consumption

*Corresponding author:

Kania Difa Parama

E-mail address:

difakania@gmail.com

All authors have reviewed and approved the final version of the manuscript.

https://doi.org/10.32539/bsm.v5i12.373

\begin{abstract}
A B S T R A C T
Background: Cancer remains a health problem in the world. Estimated 12,7 million new case and 7,6 deaths cause of cancer each year. Oral carcinoma is one of head and neck carcinoma. Oral carcinoma is 6 th the most cancer in the world and $90 \%$ of the cases are squamous cell carcinoma. The purpose of this research is to study the clinic-epidemiological profile among Indonesian patients associated with oral cancer cases managed in Head and Neck Surgery Unit of Dr Soetomo General Hospital between January 2013- December 2016. Methods: Records of 116 cases were reviewed for clinical details, histopathological data, and follow-up status. Study variables included demographic factor, modifiable risk factors such as tobacco usage and alcohol consumption, site involved, staging, histopathological result, and treatment modalities. Data was presented in form of percentages and proportions. Results: Out of the total 116 cases, majority of the subjects were above 40 years old. Twenty-two $(22 \%)$ of subjects were young adult (below 40 years old). Seventy-seven subjects $(77 \%)$ were male. It was observed that 16 cases $(21 \%)$ of male subjects was a smoker before they had disease. No female subject consumed tobacco in all form or drinking alcohol. The most common site involved was tongue, followed by lower ginggivo-buccal complex. Histopathologically, 110 cases (95\%) was welldifferentiated squamous cell carcinoma. Most of the cases $(88.8 \%)$ were in advanced stage (stage III or IV) and were treated with surgery followed by adjuvant therapy. Conclusions: The most common site for oral cancer was tongue and majority of the cases were well-differentiated squamous cancer presented in advanced stage of disease. We observed that alcohol consumption and smoking did not have any role in causing oral cancer in women, therefore further study needed to evaluate other possible factors such as diet pattern and oral hygiene. Cigarette smoking might be the cause of oral cancer in male patients.
\end{abstract}

\section{Introduction}

Currently, cancer has become a major public health problem worldwide, with an estimated number of new cases added to 12.7 million people, and causing death in 7.6 million people, or 21,000 deaths per day. In developed countries, this disease has become the number one killer, causing 2.8 million deaths per year, while in developing countries it is the second killer after heart disease with 4.8 million deaths per year. By 2030, new cancer patients worldwide are estimated to reach 21.4 million with 13.2 million deaths per year.

Carcinoma of the oral cavity is a group of malignancies that fall into the category of head and neck malignancies. The topic of discussion of oral carcinoma is almost identical to the discussion about oral squamous cell carcinoma (oral squamous cell carcinoma) because it covers $90 \%-95 \%$ of all types of malignancies. the sixth most common cancer in men after lung, prostate, colorectal, stomach, and bladder cancer. Meanwhile, in women, this cancer is in the tenth position after breast, colorectal, lung, stomach, uterine, cervical, ovarian, bladder and liver cancers. In 2002, oral cancer patients worldwide were estimated at 274,000, In the United States in 2005, there were 30,000 new cases. 
More than $90 \%$ of oral cavity carcinoma (OCC) is squamous cell carcinoma, and it is still a prominent public health problem because of the low 5-year life expectancy, ranging from 50\%.6,7 In the last 30 years, the life expectancy of people with OCC has not changed much although There have been many advances in surgical techniques, the discovery of anti-cancer drugs and radiotherapy. ${ }^{8}$ This is because many patients present at an advanced stage, even though the prognosis of this disease is closely related to the stage at which the disease is treated. Although we knew there is health insurance, prevention programs, and modern medicine, research in the United States shows that patients with oropharyngeal cancer are relatively constant, even those who come in the early stages are slightly decreased, and conversely, those who come in advanced stages increase. 1,3

The main therapy for oral carcinoma is surgery, while other therapies are additional therapies such as radiotherapy, chemotherapy, photodynamic therapy, immunotherapy and targeted therapy. Carcinoma of the oral cavity if it treated at an early stage has a survival rate of around $80-90 \%$, but in general patients come already at an advanced stage, which is approximately $53.16 \%$ of all cases. The mortality rate in advanced stages is very high, where the number of those who die in the first 5 years after being diagnosed is $50 \%-61 \%$ or a relative 5 -year survival rate of $36.3 \%$ at stage III and $23.3 \%$ at stage IV. This situation occurs both in developing countries and in developed countries.1,4 This research was aimed to study the clinical epidemiological profile among patients in
Indonesia related to the management of oral carcinoma cases in the field of head and neck surgery between January 2013 and December 2016.

\section{Methods}

Conducting a retrospective analysis by collecting data from medical records and special sheets for patients treated in the Head - Neck Surgery section of Dr. Sutomo General Hospital, with a diagnosis of oral cancer for 4 years from 2013 to 2016. A total of 116 cases were collected from medical records, histopathological data, and status review. The study variables included demographic factors, modifiable risk factors such as smoking, alcohol consumption, affected location, staging, histopathological results and treatment modalities. Data is presented in percent and proportion.

\section{Results}

Conducting a retrospective analysis by collecting data from medical records and special sheets for patients treated in the Head - Neck Surgery section of Dr. Sutomo General Hospital, with a diagnosis of oral cancer for 4 years from 2013 to 2016. A total of 116 cases were collected from medical records, histopathological data, and status review. The study variables included demographic factors, modifiable risk factors such as smoking, alcohol consumption, affected location, staging, histopathological results and treatment modalities. Data is presented in percent and proportion.

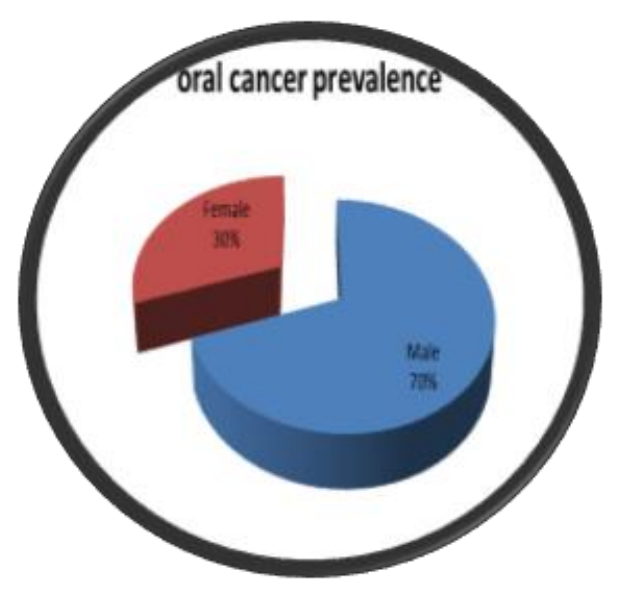

Figure 1. Prevalence diagram of oral cancer incidence in RSDS 2013-2016 


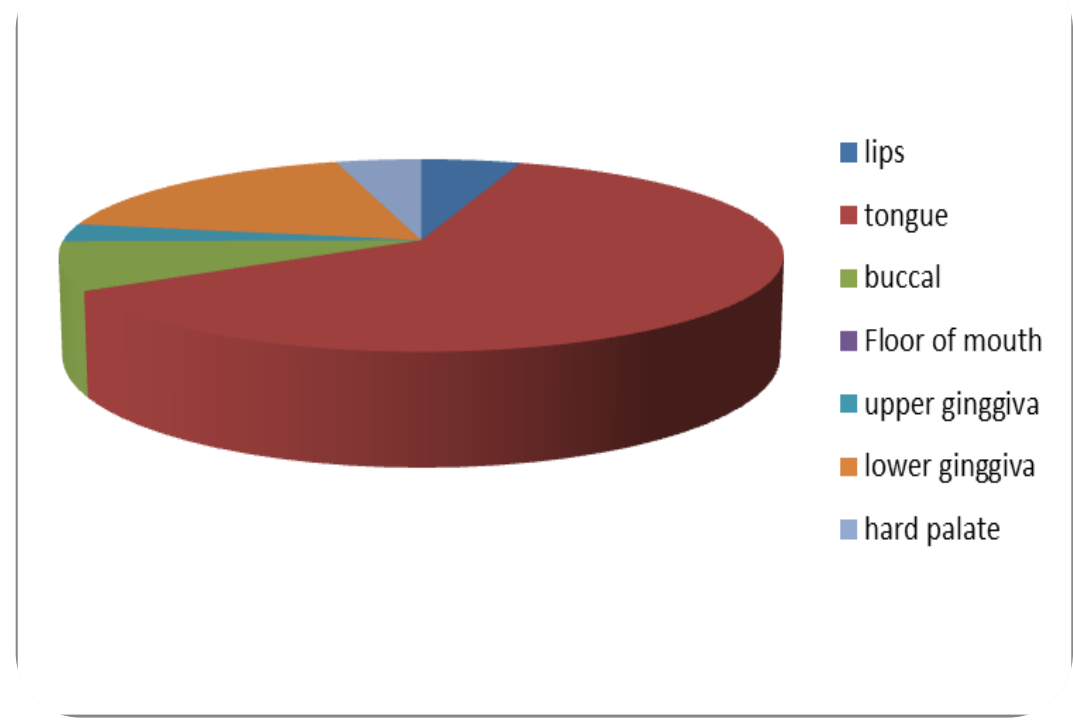

Figure 2. diagram of the most common occurrences in oral carcinoma.

Data obtained from 135 patients with clinical stage of TNM and diagnosis. based on the histopathological confirmation of the diagnosis of squamous cell carcinoma of the oral cavity were carried out at Dr. Soetomo Hospital, Department of Head and Neck Surgery from January 2014 to December 2016. histopathological appearance (table 1). From January 2014 to December 2016, 135 cases of squamous cell carcinoma were found, $70 \%$ male patients and 41 $(30 \%)$.

Data were taken from age, gender, affected site, habits,

Table 1. Distribution of oral malignancies by age

\begin{tabular}{ccc}
\hline Range of Age (in years) & Patients (n) & Percentage (\%) \\
\hline $11-20$ & 0 & 0 \\
$21-30$ & 7 & 5 \\
$31-40$ & 23 & 24 \\
$41-50$ & 32 & 34 \\
$51-60$ & 45 & 12 \\
$61-70$ & 16 & 8 \\
More than 70 & 12 & $\mathbf{1 0 0}$ \\
\hline
\end{tabular}

Based on the age distribution, most of the patients were reported to be in the age range of 51 years to 60 years, and there were no patients under the age of 30 years in this study. Judging from personal habits, patients with smoking habits are only reported about $15 \%$. Most of them smoked less than 1 pack per day and stopped smoking after there were early symptoms of the disease (figure 3). Based on the degree of histopathology, the most common is squamous cell carcinoma which is well differentiated (well differentiated 93\%) (figure 4). Most of the patients present in an advanced stage (stages II and IV). Generally comes with complaints of sores on the tongue followed by a lump on the neck (table 2). 


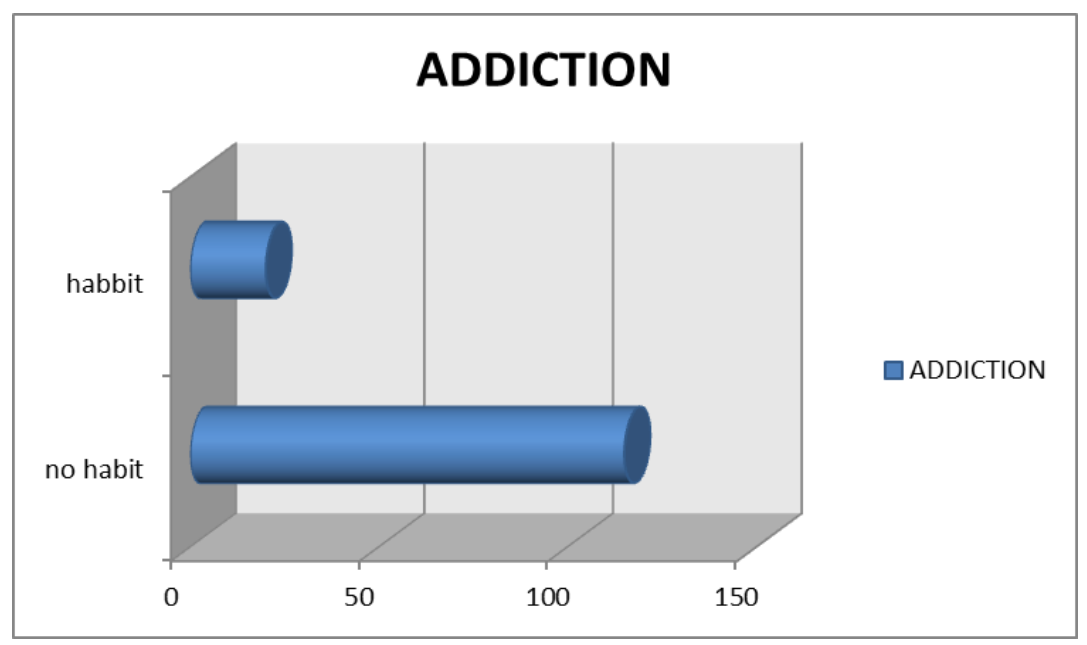

Figure 3. The percentage of smoking habit in patients.

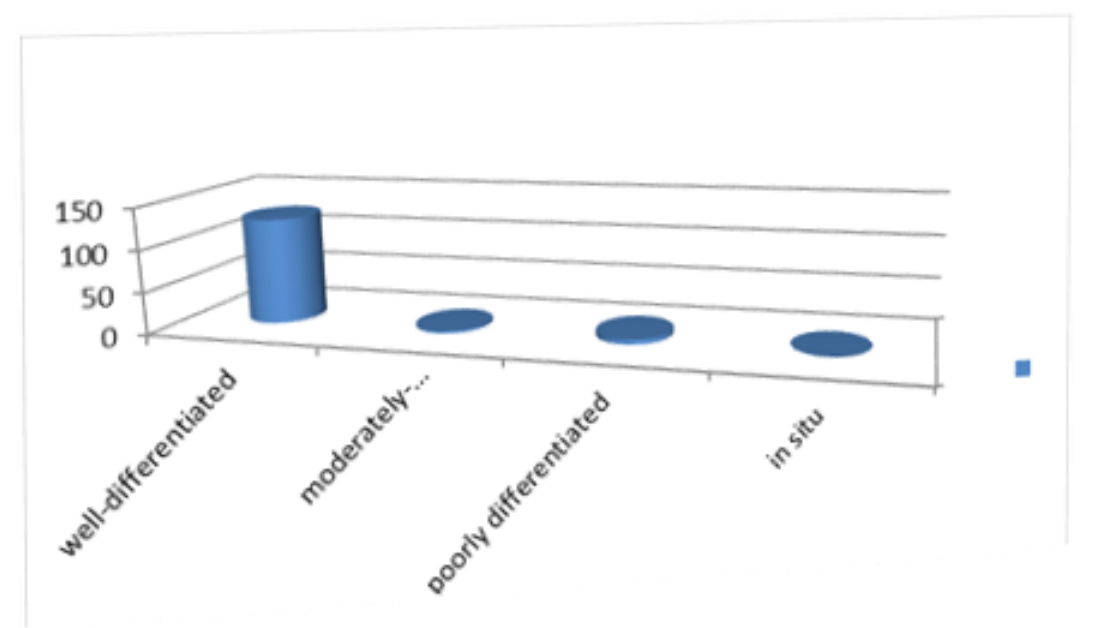

Figure 4. The degree of histopathology.

Table 2. Cancer staging and age presentation

\begin{tabular}{lcc}
\hline Staging & No of patients & Percentage (\%) \\
\hline I & 17 & 13 \\
II & 10 & 7 \\
III & 27 & 20 \\
IV & 81 & 60 \\
\hline
\end{tabular}

\section{Discussion}

The occurrence of squamous cell carcinoma of the oral cavity is a multifactorial and multistage process. This includes extrinsic factors, namely environmental factors and intrinsic factors, namely genetics. There is no single causative ingredient or factor that can be determined as a carcinogenic substance, but both extrinsic and intrinsic factors work together. Extrinsic factors that play a role in the occurrence of oral cancer are tobacco, chewing betel nut, alcohol, malnutrition, sunlight, trauma, and irritation of the edges of sharp teeth or dentures, poor oral hygiene. In addition to the above factors, the human papilloma virus is suspected as the cause of squamous cell carcinoma. Genetic basis is an intrinsic factor that plays a role in the formation of squamous cell carcinoma. Loss or altered control of the cell cycle appears to be a determining factor in the development of cancer. ${ }^{1,2}$

Squamous cell carcinoma of the tongue is common 
in men and reaches its peak in the sixth and seventh decades. According to other literature, the majority of patients with oral malignancies are men, although the incidence of tongue malignancies in women is increasing progressively in the USA to reach $47 \%$. According to the distribution of locations in the oral cavity, the tongue is the location of the most common squamous cell carcinoma, about 35\%, followed by the floor of the oral cavity $30 \%$, mandibular gingiva $15 \%$, buccal mucosa 10\%, maxillary gingiva 5\%, hard palate $3 \%$, and $2 \%$ retromolars. In other literature, the incidence of squamous cell carcinoma of the tongue is between $25-40 \%$ of carcinomas of the oral cavity. From different literatures it is said that the tongue and the floor of the oral cavity are the most common locations for squamous cell carcinoma in Western countries. However, in countries where people chew tobacco and betel nuts a lot, the retromolar trigone and buccal mucosa are the most common sites for this carcinoma. 1,3

The rate of development of carcinoma of the tongue can be classified according to the TNM classification system issued by the AJCC (American Joint Committee On Cancer. TNM (T: Primary Tumor, N: Lymph Node, and M: Metastases). The highest incidence of squamous cell carcinoma metastases to regional lymph nodes according to TNM classification are NO (50\%), N1 \& N2 (20\% each), and N3 (10\%).4,5

Therapeutic management depends on the stage. Treatment for these tumors includes surgery, radiation, chemotherapy or a combination. Surgical therapy can be in the form of hemiglosectomy, wide excision and reconstruction. The choice of this surgical method depends on the size of the lesion, the location of the lesion and the metastases that occur. An understanding of the epidemiology and risk factors of oral malignancies can help in the early identification and prevention of patients with oral malignancies. Early diagnosis of oral malignancy is very important for early therapy so that the prognosis can be better. Delay in diagnosing and detecting can increase the morbidity and mortality of the patient. The distribution of the male-female ratio in this study of 2.3:1 is similar to that reported in most other studies, Lype et al., also reported a male-to-female ratio (2.3:1). 6

Most of the patients with oral squamous cell malignancy in this study were in the age range of 51 60 years, which correlates with research from Gangane et al (2007), which reported that patients with oral squamous cell malignancy were in the age range of 50 to 59 years. Based on cancer statistics, oral cancer is a major problem in India and accounts for $50-70 \%$ of all cancer diagnoses compared to the UK and USA as much as $2-3 \%$. The age incidence of oral cancer in India is highest in the world, in men 6.2/100000 and in women 4.6/1000009. Approximately 90\% of oral cancer cases in Southeast Asia are associated with chewing tobacco and smoking. ${ }^{7}$

In our study, the tongue was the most common site of oral squamous cell malignancies followed by the buccal and lower gingival mucosa. Wide excision and mandibulectomy are common procedures used in patients with oral squamous cell malignancies. 126 cases were found to be well differentiated, followed by moderately differentiated and poorly differentiated carcinoma. Whereas in situ carcinoma has never been reported before. The government's program on awareness of malignancy continues to be improved by reporting a large number of cases of malignancy with well-differentiated carcinomas and various other programs. 8

Delay in diagnosing oral squamous cell malignancies is also associated with patient delays in examining the disease, delays from professionals or both, and the assumption that tumor size has not been detected. This can support the fact that poverty, illiteracy and the possibility of alternative medicine are patient delays. In this study, most of the patients reported coming in stage III and IV. As quoted from previous research, Sahudi reported, the prognosis of oral cancer is strongly influenced by the stage at which the patient received treatment. Unfortunately, the majority of oral cancer patients at Dr. Sutomo Hospital come in an advanced stage. Stage 4: 63.4\%; stage 3: $25.5 \%$; stage $2: 9.8 \%$, and stage 1 only $1.3 \%$. Slightly different from that obtained two decades ago at $\mathrm{Dr}$ Sutomo Hospital, where Murtedjo et al reported stage 4: $28.9 \%$; stage 3: $31.58 \%$; stage $2: 29.0 \%$, and stage 
1: $10.52 \%$. For overseas, Durazzo et al from Brazil get OCC stage 4: $51.3 \%$; stage 3: $11.1 \%$; stage 2: $23.2 \%$; stage 1: $13.8 \%$, and stage 0: $0.75 \%$. While Han S et al from China got stage 4: $25.2 \%$; stage 3: $22.6 \%$; stage 2: $32.5 \%$; stage 1: $19.7 \%$. Compared to the period 2007-2011 at Dr Sutomo General Hospital, it can be seen that the number of OCC patients who came in stage 4 increased significantly. This happens because of the tiered BPJS patient referral system where radiation and chemotherapy treatment can only be done in type A hospital. 9

Oral cancer should be a lesion that is easily visible and often causes problems such as pain, bleeding, or bad breath. With this fact, more patients should come at an early stage. However, in clinical practice, both in Indonesia and abroad, there are still more patients who come at an advanced stage, which in turn affects the outcome of therapy and the prognosis of the disease.

\section{Conclusion}

The most common site of oral carcinoma is the tongue and most cases are well-differentiated squamous malignancies that are in advanced stages. From the observation that alcohol and smoking do not have any role in causing oral malignancy in women, therefore further research is needed to evaluate other possible factors such as dietary patterns and oral hygiene. Smoking may be the cause of oral malignancy in male patients.

\section{References}

1. Watkinson JC. Stell \& Maran's Head \& Neck Surgery.4th ed. Oxford: Butterworth Heinemann. 2000; 450-65.

2. Cummings CW. Otolaryngology - head and neck surgery. 2nd ed. St Louis: Mosby Year book, 1993; 1248-70.

3. Shah JP. Head and neck surgery and oncology. 3rd ed. USA: Mosby. 2003; 333-90.

4. Neville BW. Oral \& maxillofacial pathology. Philadelphia: WB Saunders Company. 2002; 356-366.

5. American Joint Committee on Cancer. AJCC cancer staging manual. 5th ed. Philadelphia:
Lippincott-Raven. 1997; 24-26.

6. Reksoprawiro, S. Protokol penatalaksanaan kanker rongga mulut. Protokol PERABOI, 2003; 49-72.

7. Wood NK, Goaz PW. Differential diagnosis of oral and maxillofacial lession. USA: Mosby. 1997; 588.

8. Salim S. Penanganan kanker rongga mulut di RSUD Dr. Sutomo. Klinikostatistik. 2007.

9. Agrawal $\mathrm{KH}$, Rajderkar SS. Clinicoepidemiological profile of oral cancer : A hospital based study. Indian Journal of Community Health. 2012. 24(2): 150-3. 